\title{
Analysis of Wetting Behavior and Solidification Process of Molten Urea on a Superhydrophobic Surface and its Application in Large Granular Urea Production
}

Xiaoqian Deng, Rongjie Xu, Chao Yang, Ji Li, Yuanlong Wang, Pan Wu, Changjun Liu, Houfang Lu, Wei Jiang*

Low-Carbon Technology and Chemical Reaction Engineering Laboratory, School of Chemical Engineering, Sichuan University, Chengdu, 610065, PR China
(1): Xiaoqian Deng
E-mail: 1434488434@qq.com
(2): Rongjie $\mathrm{Xu}$
E-mail: 1556681205@qq.com
(3): Chao Yang
E-mail: 1352128382@qq.com
(4): Ji Li
E-mail: lijiscu@scu.edu.cn
(5): Yuanlong Wang
E-mail: 905625766@qq.com
(6): Pan Wu
E-mail: panwu@scu.edu.cn
(7): Changjun Liu
E-mail: liuchangjun@scu.edu.cn
(8): Houfang Lu
E-mail: luhouf@163.com
(9): Wei Jiang
E-mail: weijiang@scu.edu.cn

\footnotetext{
${ }^{*}$ Corresponding author. Tel.: +86-28-85990133; Fax: +86-28-85460556

E-mail: weijiang@scu.edu.cn
} 


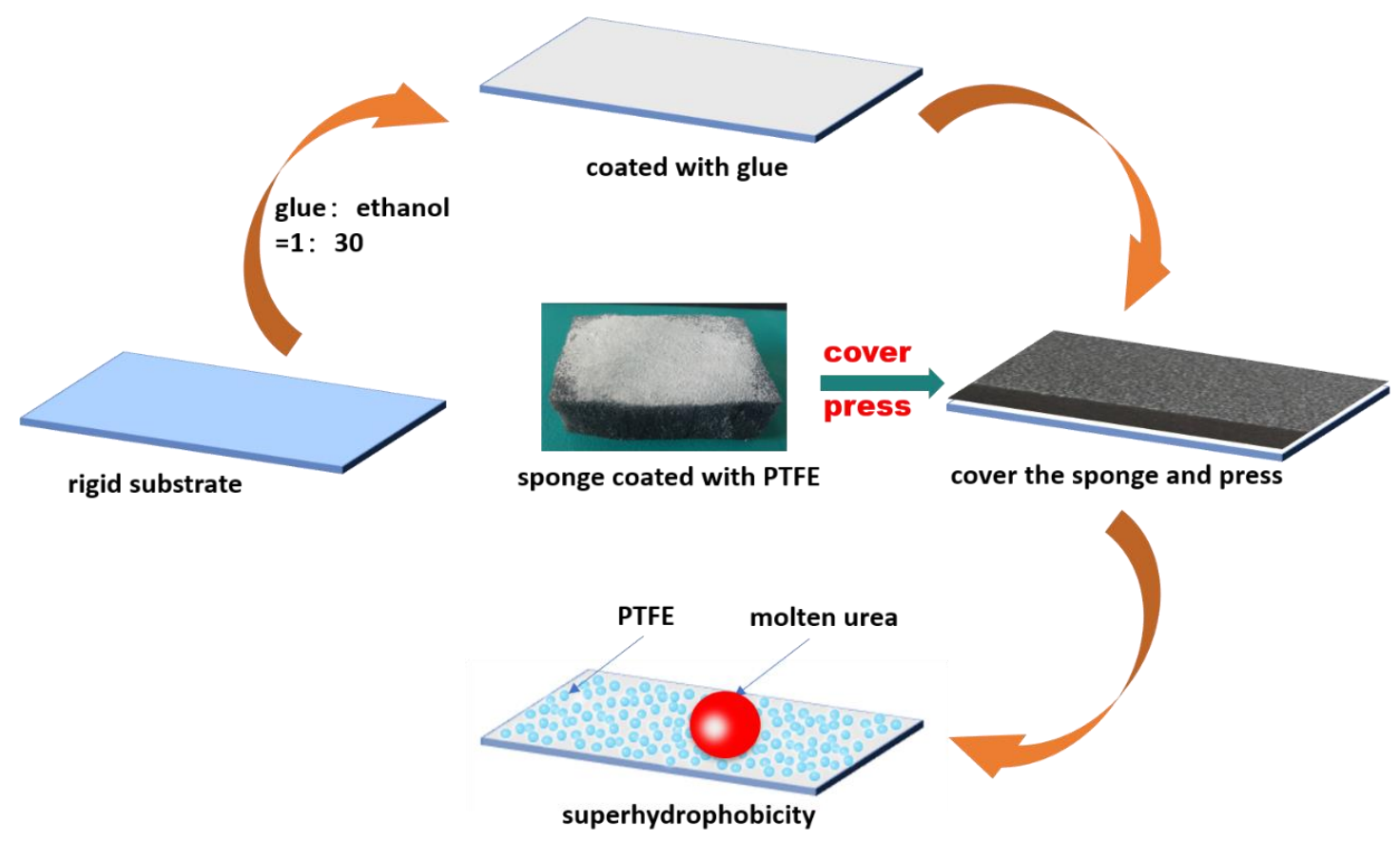

Scheme S1. Diagram of preparation of PSSSS

A simple adhesion method by pressing AU spouge dipping with PTFE nanoparticles on stainless steel surface with glue layer, can get PTFE- coated superhydrophobic stainless steel surface(PSSSS), which have super-repellence property to urea melt.

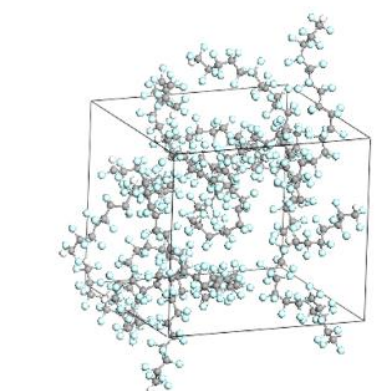

PTFE

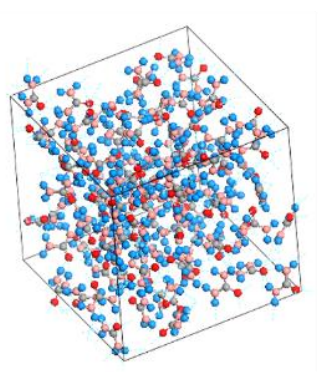

urea

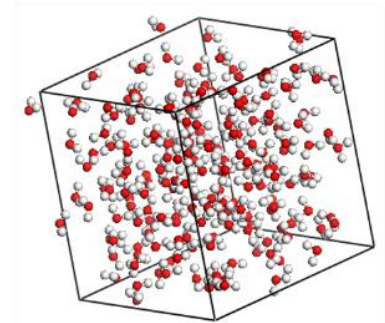

water

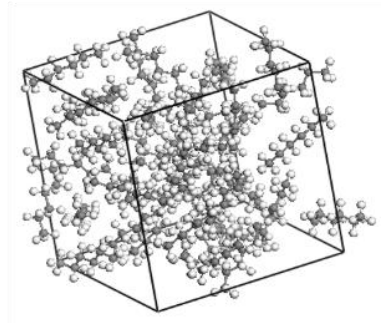

N-hexane

Figure S1. Amorphous molecular model of PTFE, urea, water and N-hexane by

Amorphous Cells 
The main step of MD simulation includes building and geometry optimization of the initial single molecule structure, constructing the amorphous molecular model of two pure substances and the blends by Amorphous Cells, and next forcite calculation (including geometry optimization, anneal, dynamics and cohesive energy density (CED) calculation. the amorphous molecular model as shown in Figure S1.

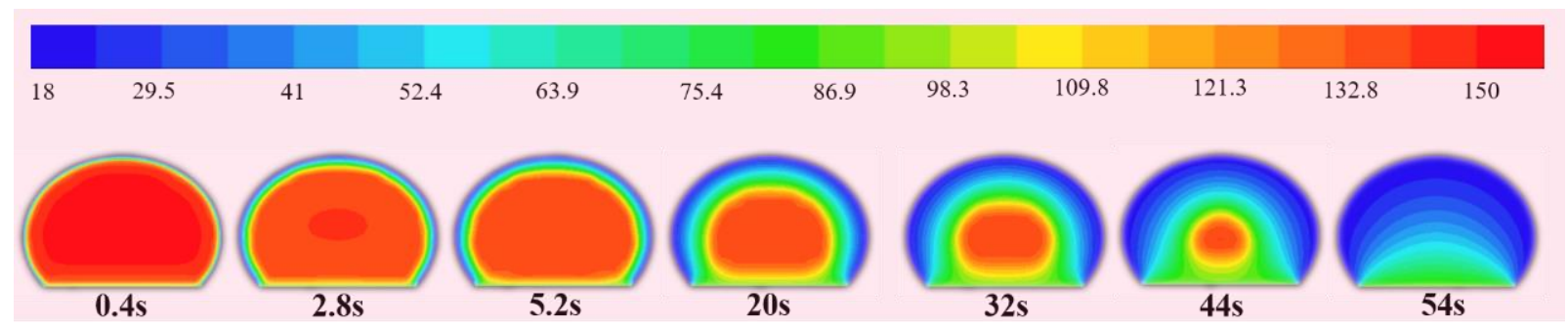

Figure S2. Solidification of urea melt on PSSSS at PSSSS temperature being $90^{\circ} \mathrm{C}$ simulate by FEM

When the PSSSS temperature is $90^{\circ} \mathrm{C}$, urea melt solidification also occur from outside to inside, although the solidfied urea shell grows slower near the bottom.

\section{EXPERIMENTAL SECTION}

Advancing contact angle. is obtained by injecting glycerin at same temperature $\left(135^{\circ} \mathrm{C}\right)$ into the urea melt drop quickly with a needle, and captured by CA meter (powereach, JC2001C1)

Receding contact angle. is obtained by pulling up the urea melt drop with a needle and capture by CA meter. 